\title{
Microscopic description of thermodynamic volume in extended black hole thermodynamics
}

\author{
Clifford V. Johnson* \\ Department of Physics and Astronomy, University of Southern California, \\ Los Angeles, California 90089-0484, USA \\ Victoria L. Martin ${ }^{\dagger}$ and Andrew Svesko \\ Department of Physics, Arizona State University, Tempe, Arizona 85287-1504, USA
}

(Received 21 January 2020; accepted 6 March 2020; published 6 April 2020)

\begin{abstract}
Using the fact that theories of gravity with asymptotically three-dimensional anti-de Sitter geometries have dual descriptions as two-dimensional conformal field theories (CFTs), we present the first study in field theory of the thermodynamic volume of various black hole solutions. We explain in general twodimensional CFT terms why the presence of a thermodynamic volume can render certain black hole solutions "superentropic." Superentropicity simply results from the fact that the Cardy formula, which gives the gravitational Bekenstein-Hawking entropy, can overcount the CFT entropy. The examples of charged Bañados, Teitelbiom, and Zanelli (BTZ) black holes and generalized exotic BTZ black holes are described. These observations help explain why the specific heat at constant volume can signal the instability of such solutions, as recently conjectured.
\end{abstract}

DOI: 10.1103/PhysRevD.101.086006

\section{INTRODUCTION}

A decade ago, a new variable was introduced into black hole thermodynamics, as part of an extended thermodynamic framework [1]. It has the interpretation as the thermodynamic volume, $V$, dual to the pressure $p \equiv$ $-\Lambda / 8 \pi$ that is present if the cosmological constant $\Lambda$ is dynamical. ${ }^{1}$ The pair $(p, V)$ joins the variables of traditional gravitational thermodynamics $[3-6],{ }^{2}$ the entropy $S=A / 4$ and temperature $T=\kappa / 2 \pi$, associated to a black hole horizon's area $A$, and surface gravity $\kappa$. The black hole's mass $M$ becomes identified with the enthalpy $H=U+p V$, where $U$ is the internal energy. (Here, we

\footnotetext{
johnson1@usc.edu

victoria.martin.2@asu.edu

*andrew.svesko@asu.edu
}

${ }^{1}$ This paper will not be concerned with the origin of the dynamics of $\Lambda$. It can be achieved in various ways if the gravity sector is embedded within a larger framework. An example is when there are also dynamical scalars $\varphi_{i}$ with a potential $V\left(\varphi_{i}\right)$. Fixed points of the potential, where the scalars take on fixed values $\varphi_{i}^{\mathrm{c}}$, give a nonzero constant $V\left(\varphi_{i}^{\mathrm{c}}\right)$, determining the value of $\Lambda$ in anti-de Sitter vacua of the theory. See e.g., the review in Ref. [2].

${ }^{2}$ For a recent historical overview, see e.g., Ref. [7].

Published by the American Physical Society under the terms of the Creative Commons Attribution 4.0 International license. Further distribution of this work must maintain attribution to the author(s) and the published article's title, journal citation, and DOI. Funded by SCOAP. are using geometrical units where $G, c, \hbar, k_{\mathrm{B}}$ have been set to unity.) The first law of thermodynamics for black holes then becomes

$$
d H=T d S+V d p
$$

(assuming for the moment that no other dynamical quantities, like charge and angular momentum, are in play). The volume is a derived quantity, calculated after the mass [hence enthalpy $H(S, p)$ ] of the solution has been identified: $V \equiv \partial H /\left.\partial p\right|_{S}$. In the simple case of static black holes (with no additional nontrivial scalar sector) it has the geometric interpretation as the naive spherical volume occupied by the black hole, but in general it is nongeometrical $[8,9]$. In such general settings, it becomes a truly independent variable from the entropy, and the physics associated with it becomes richer.

In the context of negative $\Lambda$, where the gravitational physics can often be recast in terms of a dual (nongravitational) field theory in one dimension fewer using the correspondence between anti-de Sitter dynamics and conformal field theory physics (the AdS/CFT correspondence) [10-13], it is natural to ask whether $V$ has a direct interpretation in the field theory. ${ }^{3}$ In general, this question is rather hard to explore, since the duality addresses the strongly coupled field theory regime, which is not always

${ }^{3}$ See Refs. [1,14,15] for early ideas and remarks, and Refs. [16,17] for some explorations. 
easily accessible in traditional field theory terms. Moreover the finite $T$ regime of the AdS/CFT duality is (in general) rather less well robustly explored than the $T=0$ sector.

In the present work, we point out that progress can be made in the case of three dimensional gravity (with $\Lambda<0$ ), since in that case the duality's dictionary is rather stronger: Asymptotically anti-de Sitter geometries in three dimensions $\left(\mathrm{AdS}_{3}\right)$ are dual to conformally invariant two dimensional field theories, which are very tightly constrained in their structure. Moreover, the finite temperature $T$ is simply the (inverse) period of a cycle in the two dimensional Riemann surface the theory is defined on. We will be able to write (in Secs. II and III) the thermodynamic volume $V$ in terms of quantities very familiar in the CFT. With that achieved, it is then straightforward to translate any conditions involving $V$ into statements in the CFT.

For example, it is natural in thermodynamics to ask questions about the fixed volume sector. However, in general, ${ }^{4}$ this is somewhat mysterious from the black hole thermodynamics perspective-fixed pressure is more natural there since that is simply fixed $\Lambda$-but with a microscopic dual field theory identification such as the one presented here, progress can be made in examining the physics of the fixed volume sector. (This may be of use in furthering recent work [18-20] that has uncovered novel and potentially useful physics in the fixed volume sector of black hole thermodynamics.)

We make such progress by arriving, in an important example, at a microscopic connection between the thermodynamics of the fixed volume sector and a phenomenon called "superentropicity" identified for a class of black holes [8]. These are black holes with volume $V$ that have an entropy $S$ that exceeds the amount that a Schwarzschild black hole with the same volume would have. It was noticed in Ref. [20] that several solutions for which this is the case have a negative specific heat at constant volume, $C_{V}$, signaling an instability. For one example, the charged Bañados, Teitelbiom, and Zanelli (BTZ) solution, this could be demonstrated analytically, and a connection between the superentropicity and the negativity emerged (reviewed below). This led to a more general conjecture that superentropicity implies the $C_{V}<0$ instability.

While we do not prove the conjecture here, we find a microscopic phenomenon that seems to explain (or at least herald) the superentropicity on the gravity side, and it emerges precisely as a result of our microscopic identification of the thermodynamic volume $V$ and as a consequence of working in the fixed $V$ sector. It works as follows: The standard (microscopic) CFT expression for the entropy, $S$, of the black holes which successfully

\footnotetext{
${ }^{4}$ For static black holes with no scalars, $V$ and $S$ are not independent and so in those simple cases fixed $V$ is simply the fixed area. For most cases however, $V$ is a nongeometrical quantity independent of $S$.
}

reproduces [21-24] the gravitational Bekenstein-Hawking entropy, is usually the Cardy formula $[25,26]$ in these dualities, and it turns out to be built out of some of the same quantities as the thermodynamic volume $V$. What we show is that working at fixed, positive $V$ places a condition on the CFT sector meaning that the (naive) Cardy formula overcounts the entropy in the CFT. This is the microscopic herald of the fact that the gravity entropy (as counted by Cardy) is, in a precise sense, "too much." This is presented in detail in Secs. III and IV.

As a simple first check of our microscopic formalism and our assertion that superentropicity is connected to the overcounting seen in the CFT, we study a rather large family of examples in Sec. V. These "generalized exotic" BTZ black holes [27-29] have a rich extended thermodynamics [30] with $C_{V} \neq 0$ that can also be written in two dimensional CFT terms. While there are sectors that have negative specific heats (both $C_{p}$ and $C_{V}$ can be negative for some ranges of parameters, and positive for other ranges), these examples, which are nonunitary in some cases, are not superentropic. ${ }^{5}$ In the spirit of our methods, the thermodynamic volume $V$ can be written in terms of CFT quantities. Doing so, we see that working at fixed $V$ does not result in the Cardy formula overcounting the CFT entropy. This therefore fits with our suggestion that superentropicity is heralded by such an overcount at fixed $V$.

In Sec. VI we discuss the results further, including ideas and prospects for extending this microscopic success to higher dimensions.

\section{CFT AND STANDARD BTZ}

Two principal quantities in two dimensional conformal field theory are the energy $E$ and the spin $J$, which are given in terms of the sum and difference of the eigenvalues, $\Delta, \bar{\Delta}$, of $L_{0}$ and $\bar{L}_{0}$, the zeroth components of the right and left Virasoro generators (which define the conformal algebra):

$$
E=\frac{\Delta+\bar{\Delta}}{\ell}, \quad J=\Delta-\bar{\Delta} .
$$

Here $\ell$ is a length scale set by the cosmological constant of the dual gravity theory via $\Lambda=-1 / \ell^{2}$. The right and left Virasoro algebras have central charges $c_{R}$ and $c_{L}$, which are proportional to $\ell$. Their precise values are example dependent, as we shall see. The values of $E$ and $J$ are computed in the dual gravity theory quite readily, and are the mass $M$

\footnotetext{
${ }^{5}$ Here we disagree with the interpretation of Ref. [30]. They use a definition of superentropic inherited from the geometrical formula of Ref. [8] that focuses on area $A$, and not entropy, $S$. They therefore conclude that there is a problem with the conjecture connecting superentropicity to negative $C_{V}$ since they can find regions with positive $C_{V}$. However, we are (as is Ref. [20]) using the entropy-focused interpretation of the term superentropic as opposed to the (less physical) area-focused usage. See Secs. IV and V for more discussion.
} 
and angular momentum $J$ of the black hole spacetime. The entropy on the gravitational side is computed using the Bekenstein-Hawking formula, the quarter of the area of a horizon. (Note that "area" here will mean the circumference of a circle, since there are only two spatial dimensions in the gravity theory. There may be contributions from more than one horizon, as we shall see in later examples.) On the field theory side, this entropy is reproduced in the field theory using [21-24] the Cardy formula for the asymptotic degeneracy of states with a given conformal dimension:

$$
S=\log (\rho(\Delta, \bar{\Delta}))=2 \pi \sqrt{\frac{c_{R} \Delta}{6}}+2 \pi \sqrt{\frac{c_{L} \bar{\Delta}}{6}} .
$$

Crucially, this formula's validity depends upon the key assumption that the lowest $L_{0}, \bar{L}_{0}$ eigenvalues vanish [31]. We will revisit this issue shortly.

For the examples discussed in this paper, the spacetime metric will be of the leading BTZ $[32,33]$ form:

$$
d s^{2}=-f(r) d t^{2}+f(r)^{-1} d r^{2}+r^{2}\left(d \varphi-\frac{4 j}{r^{2}} d t\right)^{2}
$$

with $f(r)=-8 m+\frac{r^{2}}{\ell^{2}}+\frac{16 j^{2}}{r^{2}}+\cdots$

(with one exception we will discuss separately). The black hole has an outer and inner horizon, at radii denoted $r_{ \pm}$, which are the larger and smaller roots of $f(r)=0$. Depending upon the parent gravity theory in question (examples below), the parameters $m$ and $j$ determine the black hole mass $M$ and angular momentum $J$ either directly or in linear combination. The classic BTZ example has $f(r)$ as written (no extra terms) and $M=m$ and $J=j$, and together with $S$ they are

$$
M=\frac{r_{+}^{2}+r_{-}^{2}}{8 \ell^{2}}, \quad J=\frac{r_{+} r_{-}}{4 \ell}, \quad S=\frac{\pi r_{+}}{2} .
$$

Comparing the first two quantities to those in Eq. (2) gives, after a little algebra:

$$
\Delta=\frac{\left(r_{+}+r_{-}\right)^{2}}{16 \ell}, \quad \bar{\Delta}=\frac{\left(r_{+}-r_{-}\right)^{2}}{16 \ell} .
$$

Using these in Eq. (3) with $c_{R}=c_{L}=3 \ell / 2$ yields the gravity entropy in Eq. (5).

In extended thermodynamics, the pressure is given by $p=1 / 8 \pi \ell^{2}$, and the mass $M$ is the enthalpy

$$
H(S, p)=4 \pi p\left(\frac{S}{\pi}\right)^{2}+\frac{\pi^{2} J^{2}}{2 S^{2}} .
$$

We will work at fixed $J$ henceforth, treating it as a parameter. The first law remains as in Eq. (1). Hence, the thermodynamic volume and temperature turn out to be

$$
\left.V \equiv \frac{\partial H}{\partial p}\right|_{S}=\pi r_{+}^{2},\left.\quad T \equiv \frac{\partial H}{\partial S}\right|_{p}=\frac{r_{+}^{2}-r_{-}^{2}}{2 \pi \ell^{2} r_{+}},
$$

the latter agreeing with either a surface gravity computation or the requirement of regularity of the Euclidean section [34].

We can go a step further. The CFT/gravity relations (6) can be inverted to give $r_{ \pm}$in terms of $\Delta$ and $\bar{\Delta}$, and so we can write $V$ in terms of CFT quantities as

$$
V=\frac{8 \pi}{3}\left(\sqrt{c_{R} \Delta}+\sqrt{c_{L} \bar{\Delta}}\right)^{2} .
$$

We propose that this relationship should be read in an analogous manner to how the Cardy formula in Eq. (3) is read. States can be constructed in the CFT in the usual manner, acting on the vacuum with the left and right (negatively moded) Virasoro generators as creation operators. Then $L_{0}$ and $\bar{L}_{0}$ measure $\Delta$ and $\bar{\Delta}$. For given values of these quantities, Eq. (9) defines a quantity $V$ that has the interpretation as the thermodynamic value in the gravity theory. Since it is made from (the square of) the same combination of CFT quantities that $S$ is built from, there is not much more to learn from this example. Questions about $V$ are equivalent to questions about $S$, as they are not independent quantities.

\section{CHARGED BTZ BLACK HOLES}

Our first example where something new arises is the charged BTZ black hole with no angular momentum, a solution of Einstein-Maxwell in three dimensions [35]. Now, we have $J=0$ and the metric function to use in Eq. (4) is instead $f(r)=-8 M+\frac{Q^{2}}{2} \log (r / \ell)+r^{2} / \ell^{2}$, where $Q$ is the $U(1)$ charge of the solution and $M$ is the mass. There is also a gauge field $A_{t}=Q \log (r / \ell)$. From the point of view of the two dimensional CFT, $Q$ is merely a deformation parameter, a global charge, which will be kept fixed here. The extended thermodynamics gives [36]

$$
\begin{aligned}
& H=\frac{4 p S^{2}}{\pi}-\frac{Q^{2}}{32} \log \left(\frac{32 p S^{2}}{\pi}\right), \quad S=\frac{\pi}{2} r_{+}, \\
& T=\frac{8 p S}{\pi}-\frac{Q^{2}}{16 S}, \quad \text { and } \quad V=\frac{4 S^{2}}{\pi}-\frac{Q^{2}}{32 p},
\end{aligned}
$$

and the first law is again Eq. (1). The internal energy of the system is given by $U \equiv H-p V=\left(Q^{2} / 32\right) \times$ $\left[1-\log \left(32 p S^{2} / \pi\right)\right]$.

Note that the presence of the charge $Q$ introduces a $\log (r / \ell)$ term in the metric function $f(r)$. Consequently, the asymptotic symmetry group of the geometry is deformed, hiding the action [37] of the Virasoro algebra. 
Crucially, we regard Virasoro as hidden, but not absent. We propose that the conformal field theory will still have the structure that we saw in the previous example, and below we will find strong evidence in support of this.

To make Virasoro explicit requires a different approach. The boundary conditions on the metric and gauge field can be modified by enclosing the entire black hole system inside some radius $r_{0}$ and introducing a renormalized mass according to $M\left(r_{0}\right)=M+\frac{Q^{2}}{16} \log \left(r_{0} / \ell\right)$, such that the manifest asymptotic Virasoro symmetry is restored [38]. This alternative scheme rearranges the thermodynamic quantities (both traditional and extended). In the resulting extended thermodynamics (which requires promoting the scale $r_{0}$ to a dynamical variable in order to have a consistent first law [36]) the thermodynamic volume $V$ loses its $Q$ dependence, becoming the geometric volume $\pi r_{+}^{2}$, and since $S=\pi r_{+} / 2$, we have $C_{V}=0$. Hence, we will not study this renormalized scheme and instead focus our attention on the thermodynamic quantities as presented in Eq. (10), which yield an interesting case study. We will revisit the issue of the renormalized scheme in a later discussion.

Notice that $V$ and $S$ in Eq. (10) are now independent. The requirement that the temperature be positive results in the restriction $Q^{2} \leq 4 \eta$, where $\eta=32 p S^{2} / \pi$. Since $V=T S / 2 p$, this also translates into positivity of the volume $V$. The parameter $\eta$ also appears in the internal energy $U$, and requiring that $U>0$ gives $\eta \leq 1$. So, just from the gravity side, we get the bound $Q^{2} \leq 4$.

Turning to the CFT quantities, $c_{L}=c_{R}=3 \ell / 2=c$ as before, and since $J=0$ we have $\Delta=\bar{\Delta}$. The Cardy formula gives the entropy as before: $S=4 \pi \sqrt{c \Delta / 6}$, but now the thermodynamic volume $V$, written in terms of CFT quantities, is

$$
V=\frac{32 \pi c}{3}\left(\Delta-\frac{Q^{2} c}{96}\right)
$$

Positivity of $V$ (following from positivity of $T$ ) translates into a nontrivial statement: The lowest $\Delta$ can be is $\Delta_{0}=Q^{2} c / 96$. Recall that an assumption underlying the Cardy formula (3) is that $\Delta_{0}=0$. In fact, when $\Delta_{0} \neq 0$, the correct formula to use for the (logarithm of the) asymptotic density of states replaces $c$ by $c_{\text {eff }} \equiv c-24 \Delta_{0}$, resulting in (for positive $\Delta_{0}$ ) a reduction of the entropy count [31]. For us, $c_{\text {eff }}=c\left(1-Q^{2} / 4\right)$, and we recover two interesting pieces of information. The first is that the gravity entropy, which corresponds to the naive Cardy formula, overcounts the number of degrees of freedom of the theory. The second is that there is a unitarity bound of $Q^{2} \leq 4$, the same bound we obtained by independent gravity requirements that $T$ and $U$ are positive.

That we have recovered precisely the same condition on $Q$ using two very different considerations (gravity and
CFT) is strong support for our proposal for writing a microscopic/CFT formula for $V$. It also strongly suggests that we were correct to use the $\mathrm{AdS}_{3} / \mathrm{CFT}_{2}$ map for this charged black hole despite the fact that the asymptotic algebra is deformed by the presence of $Q$.

The overcounting of the entropy discovered here suggests that something is wrong with the equilibrium thermodynamics suggested by the variables in Eq. (10). We propose that it is in fact a herald of the phenomenon called "superentropicity," discussed next.

\section{SUPERENTROPICITY AND INSTABILITY}

The charged BTZ solution is the simplest example of a superentropic black hole [36]. Generally, a superentropic $d$-dimensional black hole is defined as a solution which violates the reverse isoperimetric inequality [8]:

$$
\mathcal{R} \equiv\left(\frac{(d-1) V}{\omega_{d-2}}\right)^{\frac{1}{d-1}}\left(\frac{\omega_{d-2}}{4 S}\right)^{\frac{1}{d-2}} \geq 1
$$

where $V$ is the thermodynamic volume, and $S$ is the gravitational entropy. Also, the quantity $\omega_{n}=2 \pi^{(n+1) / 2} /$ $\Gamma[(n+1) / 2]$ is the standard volume of the round unit sphere. The inequality (12) is saturated by SchwarzschildAdS black holes (BTZ in $d=3$ ), with $\mathcal{R}=1$. Black holes where $\mathcal{R}>1$ are said to be subentropic, such as Kerr-AdS [8] and STU black holes [39]. Systems with $\mathcal{R}<1$, such as the ultraspinning limit of Kerr-AdS black holes [40,41], are superentropic. In $d=3$, the rotating BTZ black hole has $\mathcal{R}=1$, while the charged BTZ hole has $\mathcal{R}<1$.

Notice that the inequality (12) is written here with $\mathcal{R}$ defined in terms of the entropy $S$ instead of the horizon area $A$, as it is written in Ref. [8]. This is because, in our view, superentropicity is a statement about the thermodynamic quantity entropy (as the title suggests) and not about the outer horizon area. ${ }^{6}$ Moreover, more general theories of gravity have an entropy that is not proportional to the outer horizon area, but may include contributions from the inner horizon. ${ }^{7}$ The next section will have such examples, and as we will see, our definition (12) will lead to a different interpretation of their entropic character than the one proposed in the recent literature [30].

It was recently observed [20] that several superentropic black holes are thermodynamically unstable, signified by a negative heat capacity $C_{V}$. It was conjectured there that superentropicity may generally imply that $C_{V}<0$, following from the fact that for a charged BTZ black hole this can

\footnotetext{
${ }^{6} \mathrm{~A}$ similar modification to the reverse isoperimetric inequality was made in Ref. [42] for black hole solutions of Horndeski theories of gravity.

${ }^{7}$ In fact, sometimes even in ordinary gravity, the entropy receives contributions from other objects. See the Taub-NUT and Taub-Bolt examples in Refs. [43-45].
} 
be verified analytically: The temperature $T$ and $C_{V}$ take the form

$$
\begin{gathered}
T=\frac{\pi V}{16 S} \frac{Q^{2}}{\left(4 S^{2}-\pi V\right)}, \\
C_{V}=-S\left(\frac{4 S^{2}-\pi V}{12 S^{2}-\pi V}\right) .
\end{gathered}
$$

The temperature is positive when $4 S^{2}>\pi V$, which is equivalent to the $d=3$ superentropicity condition $\mathcal{R}<1$. Moreover, this is precisely when the charged BTZ solution has $C_{V}<0$, i.e., it is thermodynamically unstable. (Showing that $C_{V}<0$ when $\mathcal{R}<1$ was also verified numerically in Ref. [20] for a class of ultraspinning Kerr-AdS black holes in various higher dimensions. Analytic counterparts to the above $d=3$ demonstration were not obtained however.)

Positivity of $T$ ensuring a connection between superentropicity and instability is strongly reminiscent of what we saw in the previous section, when making connections to the CFT. When the dual CFT is unitary, we may translate $c_{\text {eff }}>0$ into $4 S_{\mathrm{CFT}}^{2}>\pi V$, where $S_{\mathrm{CFT}}=4 \pi \sqrt{c_{\mathrm{eff}} \Delta / 6}$. Then, since $S>S_{\mathrm{CFT}}$, we have $4 S^{2}>\pi V$. Therefore, superentropicity reflects that the gravitational entropy overcounts the number of degrees of freedom of the underlying microscopic theory.

The overcounting is also accompanied by the negativity of $C_{V}$, which itself suggests an instability, a movement in solution space to some new set of thermodynamic quantities for which $C_{V}$ is no longer negative. It is tempting to speculate that the extended thermodynamics yielded [36] by studying the renormalized scheme of Ref. [38] [reviewed briefly below Eq. (10)] is the endpoint of the instability. One suggestion of our observations here is that there is another framework [different from the renormalization scheme recalled below Eq. (10)] in which the asymptotic Virasoro algebra is restored, but in which the central charge is modified to our effective central charge $c_{\text {eff }}=c\left(1-Q^{2} / 4\right)$. It would be interesting to find such a framework, and to see whether the resulting thermodynamic quantities produce a super- or subentropic system.

\section{GENERALIZED EXOTIC BTZ BLACK HOLES}

As a final example we consider the family of "generalized exotic BTZ" black holes [27-29]. The relevant gravity theory is a linear combination of the EinsteinHilbert action and the gravitating Chern-Simons action, $I=\alpha I_{\mathrm{EM}}+\gamma I_{\mathrm{GCS}}$, where $\gamma=1-\alpha$. The metric is again given in Eq. (4), with no extra terms for $f(r)$, but this time the mass and angular momentum mix the parameters $m$ and $j: M=\alpha m+\gamma j / \ell, J=\alpha j+\gamma \ell m$. The case of $\alpha=1$ is the standard BTZ black hole, while $\gamma=1$ is the exotic BTZ black hole. General $0 \leq \alpha \leq 1$ interpolates between these two extremes. The thermodynamic variables are given by

$$
\begin{aligned}
M & =\frac{\alpha\left(r_{+}^{2}+r_{-}^{2}\right)}{8 \ell^{2}}+\frac{\gamma r_{+} r_{-}}{4 \ell^{2}}, \\
J & =\frac{\alpha r_{+} r_{-}}{4 \ell}+\frac{\gamma\left(r_{+}^{2}+r_{-}^{2}\right)}{8 \ell}, \\
T & =\frac{r_{+}^{2}-r_{-}^{2}}{2 \pi \ell^{2} r_{+}}, \quad \Omega=\frac{r_{-}}{r_{+} \ell}, \\
S & =\frac{\pi}{2}\left(\alpha r_{+}+\gamma r_{-}\right), \\
V & =\alpha \pi r_{+}^{2}+\gamma \pi r_{-}^{2}\left(\frac{3 r_{+}}{2 r_{-}}-\frac{r_{-}}{2 r_{+}}\right),
\end{aligned}
$$

where $\Omega$ is the angular velocity.

Recently it was shown that generalized exotic BTZ solutions can have $C_{V}$ both positive and negative [30]. Specifically, for $\alpha<1 / 2, C_{V}$ is positive for large enough $r_{+}$. In the regions where $C_{V}>0$, however, the heat capacity at constant pressure $C_{p}$ will be negative, indicating that they are generally unstable. Notice that for the inequality (12), we have

$$
\mathcal{R}=\frac{1}{2(\alpha+\gamma x)} \sqrt{4 \alpha+6 \gamma x-2 \gamma x^{3}}
$$

where $x \equiv r_{-} / r_{+}$ranges between 0 and 1 . For the defined range of nonzero $\alpha$, we find $\mathcal{R}>1$, and thus these generalized exotic BTZ black holes form a class of subentropic black holes. Had we instead used the form of $\mathcal{R}$ first written in [8], we would have found $\mathcal{R}<1$ and concluded that these solutions are superentropic, as Ref. [30] does. ${ }^{8}$ However, as we have already stated, we are using the entropy-focused interpretation of the term superentropic as opposed to the (less physical) area-focused usage. In this sense, in the spirit of Ref. [20]'s conjecture and what we have seen in the previous two sections, there is no superentropicity and hence $C_{V}$ does not need to become negative, since the solution does not need to somehow shed the extra entropy.

Turning to the dual conformal field theory, some algebra shows that variables $M, J$, and $S$ fit the CFT form given in Eqs. (2) and (3) (with factors $\alpha+\gamma=1$ for right-moving quantities and $\alpha-\gamma=2 \alpha-1$ for left-moving):

$$
\begin{aligned}
\Delta & =\frac{1}{16 \ell}\left(r_{+}^{2}+r_{-}^{2}\right), \quad \bar{\Delta}=\frac{2 \alpha-1}{16 \ell}\left(r_{+}^{2}-r_{-}^{2}\right), \\
c_{R} & =\frac{3 \ell}{2}, \quad c_{L}=\frac{3 \ell}{2}(2 \alpha-1) .
\end{aligned}
$$

We may recast the thermodynamic volume $V(14)$ in terms of these CFT parameters. The resulting expression is

\footnotetext{
${ }^{8}$ We thank W. Cong and R. B. Mann for helpful communications about this matter.
} 


$$
\begin{aligned}
\frac{3 V}{4 \pi c_{R}}= & \left(1+\frac{1}{\epsilon}\right)(\sqrt{\Delta}+\sqrt{\epsilon \bar{\Delta}})^{2} \\
& +\left(1-\frac{1}{\epsilon}\right)\left(\frac{\sqrt{\Delta}-\sqrt{\epsilon \bar{\Delta}}}{\sqrt{\Delta}+\sqrt{\epsilon \bar{\Delta}}}\right)[\Delta+\epsilon \bar{\Delta}+4 \sqrt{\epsilon \Delta \bar{\Delta}}],
\end{aligned}
$$

where $\epsilon \equiv c_{R} / c_{L}$. Note that $c_{R}=c_{L}$ when $\alpha=1, \gamma=0$, i.e., we have the usual BTZ solution of Sec. II, and our expression (17) reduces to the thermodynamic volume given in Eq. (9).

The key observation from (17) is that, unlike the charged BTZ case, requiring positivity of $V$ does not lead to a shift away from zero for the lowest value of $\Delta$ or $\bar{\Delta}$. As such, the gravitational entropy (as given by the Cardy formula) does not overcount the number of microscopic degrees of freedom. This fits with the observation above that there is no superentropicity in these examples [using the entropyfocused definition of $\mathcal{R}$ in Eq. (12)].

\section{CONCLUSION}

In conclusion, we have shown how to microscopically interpret (using $\mathrm{AdS}_{3} / \mathrm{CFT}_{2}$ duality) the thermodynamic volume of extended black hole thermodynamics, by writing formulas for it in terms of CFT quantities. For simple black holes where $V$ and $S$ are not independent, such a formula is no more useful than the Cardy formula for $S$. However, deploying the interpretation in the charged BTZ example where $V$ is independent of $S$, we uncovered that the naive Cardy formula overcounts the entropy of the theory. We interpret this as a microscopic herald of the superentropicity phenomenon associated to some solutions in extended thermodynamics.

Independent conditions derived from gravity and CFT gave precisely the same bound on $Q$, the black hole charge: $Q^{2} \leq 4$, suggesting internal consistency of our methods. These methods included using the CFT dual of the charged black hole solution even though the presence of $Q$ deforms the asymptotic symmetry (Virasoro) algebra. This might suggest that there is another framework [different from the renormalization scheme recalled below Eq. (10)] in which the asymptotic Virasoro algebra is restored, but in which the central charge is modified to our effective central charge $c_{\text {eff }}=c\left(1-Q^{2} / 4\right)$. It would be interesting to find such a framework, and to see whether the resulting thermodynamic quantities produce a super- or subentropic system.

It would also be interesting to find a similar microscopic understanding of superentropicity of ultraspinning black holes [40,41]. These solutions exist for $d \geq 4$, where we can no longer use $\mathrm{AdS}_{3} / \mathrm{CFT}_{2}$ duality. Instead, however, we could consider Kerr/CFT duality [46], along the lines of Ref. [47], and see if constraints imposed by the gravitational thermodynamics lead to any requirements on the dual CFT. We leave this for future work.

Another line of investigation could be to develop further a characterization of how superentropicity may result in the $C_{V}<0$ instability for other black holes, and in other dimensions. As conjectured in Ref. [20], a consequence of superentropicity is negativity of $C_{V}$. (Note again that this is not the same as saying that negativity of $C_{V}$ implies superentropicity.) For the charged BTZ case this was shown directly in Eq. (13), where the form and sign of $C_{V}$ depends solely on the ability to write the temperature as $T=\mathcal{F}(S, V, Q) /(1-\mathcal{R})$, where $\mathcal{F}$ is a function we wish to characterize further, and the $\mathcal{R}$ in the denominator is given in Eq. (12). Not every black hole solution will have a temperature that can be written in this form, as we see in the cases of the uncharged and exotic BTZ black holes. Moreover, we know of subentropic solutions whose temperature does take this form, e.g., the $d=4$ Kerr-AdS black hole [48]. Nonetheless, we might attempt to learn something about a subclass of superentropic black hole solutions by demanding the temperature take the form given above. If they have negative $C_{V}$, it implies conditions on $\mathcal{F}$. Our special form of $T$ together with the fact that $T=f^{\prime}\left(r_{+}\right) / 4 \pi$ for a gravity solution with metric function $f(r)$ might characterize enough about the properties of $f(r)$ to use it as a diagnostic tool for a wide variety of solutions. We leave this for future work.

\section{ACKNOWLEDGMENTS}

C. V. J.'s work was supported by the U.S. Department of Energy under Grant No. DE-SC0011687. Some of this work was carried out at the Aspen Center for Physics, which is supported by National Science Foundation Grant No. PHY-1607611. The work of V.L.M. is supported by the U.S. Department of Energy under Grant No. DESC0018330. A. S. and V. L. M. thank the USC Department of Physics and Astronomy, where work on this project began, for hospitality.
[1] D. Kastor, S. Ray, and J. Traschen, Classical Quantum Gravity 26, 195011 (2009).

[2] O. Aharony, S. S. Gubser, J. M. Maldacena, H. Ooguri, and Y. Oz, Phys. Rep. 323, 183 (2000).
[3] J. D. Bekenstein, Phys. Rev. D 7, 2333 (1973).

[4] J. D. Bekenstein, Phys. Rev. D 9, 3292 (1974).

[5] S. Hawking, Commun. Math. Phys. 43, 199 (1975).

[6] S. Hawking, Phys. Rev. D 13, 191 (1976). 
[7] D. Grumiller, R. McNees, and J. Salzer, Fundam. Theor. Phys. 178, 27 (2015).

[8] M. Cvetic, G. Gibbons, D. Kubiznak, and C. Pope, Phys. Rev. D 84, 024037 (2011).

[9] C. V. Johnson, Classical Quantum Gravity 31, 235003 (2014).

[10] J. M. Maldacena, Adv. Theor. Math. Phys. 2, 231 (1998).

[11] S. S. Gubser, I. R. Klebanov, and A. M. Polyakov, Phys. Lett. B 428, 105 (1998).

[12] E. Witten, Adv. Theor. Math. Phys. 2, 253 (1998).

[13] E. Witten, Adv. Theor. Math. Phys. 2, 505 (1998).

[14] B. P. Dolan, Classical Quantum Gravity 31, 035022 (2014).

[15] C. V. Johnson, Classical Quantum Gravity 31, 205002 (2014).

[16] B. P. Dolan, J. High Energy Phys. 10 (2014) 179.

[17] J. Couch, W. Fischler, and P. H. Nguyen, J. High Energy Phys. 03 (2017) 119.

[18] C. V. Johnson, Classical Quantum Gravity 37, 054003 (2020).

[19] C. V. Johnson, Classical Quantum Gravity 37, 034001 (2020).

[20] C. V. Johnson, Mod. Phys. Lett. A 33, 2050098 (2020).

[21] S. Carlip and C. Teitelboim, Phys. Rev. D 51, 622 (1995).

[22] A. Strominger and C. Vafa, Phys. Lett. B 379, 99 (1996).

[23] A. Strominger, J. High Energy Phys. 02 (1998) 009.

[24] D. Birmingham, I. Sachs, and S. Sen, Phys. Lett. B 424, 275 (1998).

[25] J. L. Cardy, Nucl. Phys. B270, 186 (1986).

[26] H. W. J. Bloete, J. L. Cardy, and M. P. Nightingale, Phys. Rev. Lett. 56, 742 (1986).

[27] S. Carlip and J. Gegenberg, Phys. Rev. D 44, 424 (1991).

[28] S. Carlip, J. Gegenberg, and R. B. Mann, Phys. Rev. D 51, 6854 (1995).

[29] P. K. Townsend and B. Zhang, Phys. Rev. Lett. 110, 241302 (2013).
[30] W. Cong and R. B. Mann, J. High Energy Phys. 11 (2019) 04.

[31] S. Carlip, Classical Quantum Gravity 15, 3609 (1998).

[32] M. Banados, C. Teitelboim, and J. Zanelli, Phys. Rev. Lett. 69, 1849 (1992).

[33] M. Banados, M. Henneaux, C. Teitelboim, and J. Zanelli, Phys. Rev. D 48, 1506 (1993); 88, 069902(E) (2013).

[34] G. W. Gibbons and S. W. Hawking, Phys. Rev. D 15, 2752 (1977).

[35] C. Martinez, C. Teitelboim, and J. Zanelli, Phys. Rev. D 61, 104013 (2000).

[36] A. M. Frassino, R. B. Mann, and J. R. Mureika, Phys. Rev. D 92, 124069 (2015).

[37] J. D. Brown and M. Henneaux, Commun. Math. Phys. 104, 207 (1986).

[38] M. Cadoni, M. Melis, and M. R. Setare, Classical Quantum Gravity 25, 195022 (2008).

[39] E. Caceres, P. H. Nguyen, and J. F. Pedraza, J. High Energy Phys. 09 (2015) 184.

[40] R. A. Hennigar, D. Kubiznak, and R. B. Mann, Phys. Rev. Lett. 115, 031101 (2015).

[41] R. A. Hennigar, D. Kubiznak, R. B. Mann, and N. Musoke, J. High Energy Phys. 06 (2015) 096.

[42] X.-H. Feng, H.-S. Liu, W.-T. Lu, and H. Lu, Eur. Phys. J. C 77, 790 (2017).

[43] A. Chamblin, R. Emparan, C. V. Johnson, and R. C. Myers, Phys. Rev. D 59, 064010 (1999).

[44] R. Emparan, C. V. Johnson, and R. C. Myers, Phys. Rev. D 60, 104001 (1999).

[45] R. B. Mann, Phys. Rev. D 60, 104047 (1999).

[46] M. Guica, T. Hartman, W. Song, and A. Strominger, Phys. Rev. D 80, 124008 (2009).

[47] M. Sinamuli and R. B. Mann, J. High Energy Phys. 08 (2016) 148.

[48] B. P. Dolan, Classical Quantum Gravity 28, 235017 (2011). 